\title{
Mitochondrial genetics and cancer
}

\author{
Safarina G. Malik* \\ Eijkman Institute for Molecular Biology JI. Diponegoro no. 69, Jakarta 10430, \\ Indonesia
}

DOI: http://dx.doi.org/10.19106/JMedScieSup004804201628

\section{ABSTRACT}

The first modern human, the Mitochondrial Eve, was traced back to Africa about 200,000 years ago, based on the variation in the mitochondrial DNA (mtDNA). The eruption of Mount Toba super-volcano in Sumatra 70,000 years ago may have led to a 'nuclear winter', followed by a 1,000-year ice age. This cold snap would have made life difficult; genetic evidence indicated a sharp reduction in population size around this time, reaching approximately 10,000 individuals. Once the climate started to improve, our ancestors recovered from this near-extinction event. The population expanded, and some courageous explorers ventured beyond Africa. Around 50,000 years ago some of these brave ancestors had successfully crossed the globe to South East Asia and Australia. Some of them settled in the Indonesian archipelago, forming the first settlement of prehistoric Indonesia. The second migration happened around 10,000 years ago, where a group of hunter-gatherers followed the now-submerged river systems that once ran from mainland Asia between the modern islands of Sumatera, Java, and Borneo. Then, around 4,000 years ago the third group of ancestors arrived. This agricultural community brought along their culture of pottery, plant cultivation, and animal domestication, occuring in coincidence with the vast spread of Austronesian languages. Therefore, it is likely that the Indonesian archipelago hosted a wide range of linguistic, ethnic and genetic diversity. Nowadays, the modern Indonesia is home to around 700 ethnic populations, each with distinct cultural and linguistic characteristics, representing vast genome diversity.

Our ancestors' decision to embark on sea travel and take on its related lifestyle has influenced the development of susceptibility and resistance to various diseases observed today. During the prolonged travel, our ancestors were subjected to changes in global climate and geographic dynamic, which strongly influenced and shaped the genetic background of modern humans, including the mtDNA genome. Mitochondria, a well-adapted endosymbiotic intracellular organelles, became efficient for energy production throughout the course of evolution. They are critical for survival and proliferation of living organisms under aerobic conditions and produce ATP through oxidative phosphorylation (OXPHOS). Adaptation to new environments that favor beneficial traits might have caused genetic risk differences that influence the crucial function of the mitochondria, consequently affecting many functions in the cell. The altered function of the mitochondria might act as an important factor for disease susceptibility across many human populations, i.e. mtDNA variation grouped together to form a certain type/group (the mtDNA haplogroup) was reported to modulate cancer susceptibility and resistance in Chinese population.

Corresponding author: joanne.ngeow.y.y@singhealth.com.sg 
Cancer cells are characterized in general by a decrease of mitochondrial respiration and OXPHOS, a consequence of disruptive mtDNA mutations, thus one might affirm that the growth of cancer cells is directly limited by cellular energetics. In order to survive, cancer cells must modify their mitochondrial physiology to optimize energy production towards their changing environments. There are two types of advantageous mtDNA mutations for cancer cells: mutations that impair OXPHOS and serve to stimulate neoplastic transformation, and those that facilitate cancer cell adaptation to altered bioenergetics environment. These mtDNA mutations would eventually lead to an enhanced generation of reactive oxygen species (ROS), which acts both as mutagens and cellular mitogens, and contribute directly to cancer progression. Therefore, it can be concluded that mitochondrial alterations are critical for cancer initiation, promotion, and metastasis (Fig 1).

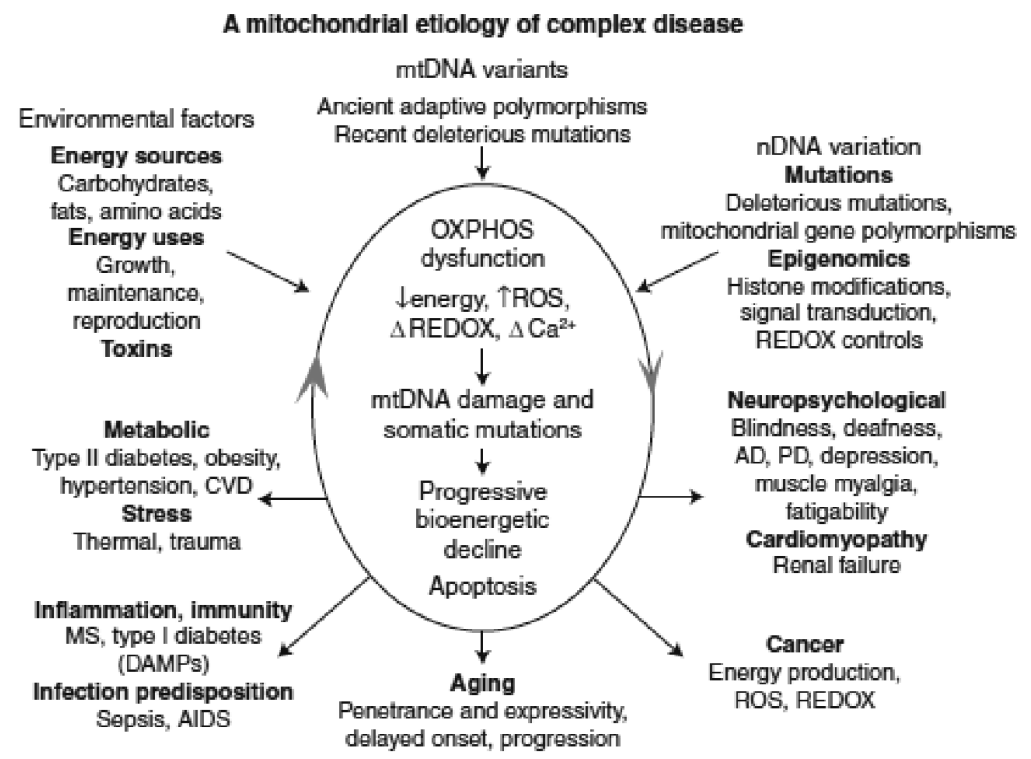

FIGURE 1. Integrated mitochondrial paradigm explaining genetic and phenotypic complexities of metabolic and degenerative disease, aging, and cancer.

Top three arrows: factors that have impact on mitochondrial OXPHOS robustness, risk for developing disease symptoms. Central oval arrows: pathophysiological basis of disease processes and the basis of disease progression. Lower five arrows: summarized disease categories and phenotypic outcomes of disturbed mitochondrial energy transformation. Bottom arrow: effect of the problematic accumulation of somatic mtDNA mutations resulting in delayed-onset and progressive course of diseases and aging. Right arrow: clinical problems that can result from reduced energy production in the most energetic tissues: the brain, heart, muscle, and kidney. Left arrow: indicates the metabolic effects of mitochondrial dysfunction, which result in the perturbation of the body's energy balance. Lower right arrow: mitochondrial alterations are critical for cancer initiation, promotion, and metastasis. Lower left arrow: the hypothesized inflammatory and autoimmune responses that may result from chronic introduction of mitochondria's bacterialike DNA and $\mathrm{N}$-formylmethionine proteins into the bloodstream. 